\title{
Design of Torque Balancing Mechanisms
}

\author{
Bruno Zappa, Vittorio Lorenzi, Paolo Righettini and Roberto Strada \\ Department of Engineering and Applied Sciences, University of Bergamo, Dalmine 24044, Italy
}

\begin{abstract}
Machines are often subjected to periodic loads, related both to the characteristics of the payload or to the kinematic chains used for motion generation, such as linkages or cam follower mechanisms. The load fluctuation can cause several inconveniences to the proper functioning of the machines, like shaking forces and moments, vibrations, severe speed fluctuations. Speed fluctuations are a main source of concern since, generally, mechanisms design and optimization is performed considering a constant main shaft speed and a departure from ideal behavior gets worse increasing machine speed. The simpler approach used to mitigate this fact consists in adopting a large flywheel and/or a massive motor, even to drive small loads. More sophisticated procedures add appropriate balancer mechanisms to the machine. This paper, starting from a review of the available literature, presents a theoretical framework to the problem of input torque balancing and defines a methodology for the synthesis of balancing mechanisms.
\end{abstract}

Key words: Torque balancing, flywheel, periodic motion.

\section{Introduction}

Machines generally contain reciprocating and/or rotating masses: when running at high speed significant inertia forces and torques can arise. These actions are transferred directly to the machine frame and then to the environment causing vibrations, resonances, noise etc. A reduction of these phenomena can be obtained through a careful isolation of the machine and/or the balancing of the shaking forces and moments. Several methods for linkage balancing have been proposed. Comprehensive surveys of shaking force and shaking moment balancing can be found in Refs. [1, 2]. Much wider is the literature regarding vibration insulation.

This paper will focus on another important effect that must be taken into account in periodic running machines: the fluctuation of the torque acting on the motor shaft resulting, generally, into speed variation. This fluctuation could be present even after complete inertia forces balancing. Generally, such machines are analyzed and designed assuming a constant speed of

Corresponding author: Roberto Strada, Ph.D., assistant professor, research fields: mechatronic systems, electric actuators, modeling and simulation of fluid power systems and components (pneumatics, hydraulics). the input shaft and hence of the motor. However, in high speed machines, the torque required to drive the input shaft at constant speed exhibits a very high periodic fluctuation, hence massive motors are required to drive even small loads. On the other hand, when the system is driven by a low stiffness motor, such fluctuations affect the required motion of the system, lowering machine performance. Moreover, in case of backlash, if the input torque frequently changes its sign, transmission gears and system joints could be not properly loaded. In all cases an increase in the machine vibration is observed. The simplest approach to reduce torque/speed fluctuations is based on the addition of a large flywheel on the "constant speed" shaft. As a drawback, a large flywheel implies slow transients (both in start and stop phases), an increase of the overall weight of the machine and, even worse, a lowering of machine natural frequencies.

This paper, starting from a review of the available literature, presents a theoretical framework to the problem of torque balancing and defines a methodology for the synthesis of balancing mechanisms with the task to fully compensate the load variations due to a periodic torque.

Several input torque balancing techniques have been developed in the literature. Input torque 
balancing can be achieved by adding to the original mechanical system a compensating device whose goal is to partly or completely eliminate the input torque fluctuation sensed by the main shaft [3,4]. Generally, such compensating devices, henceforth we call them balancers, are driven by a suitable shaft of the machine, usually the main shaft, and can be seen as energy accumulators.

This device can be designed to store/supply kinetic energy, hence the name "kinetic balancer" (KB for brevity), or potential energy (generally elastic), hereafter we call it "potential balancer" or PB for short.

Many "kinetic balancers" have been proposed, in Ref. [5] the balancer consists in a flywheel driven through a noncircular gear pair, similarly Ref. [6] uses noncircular gears to drive the rocker of a five-bar linkage that acts as the balancer. In Ref. [7] the input torque balancing is obtained using a "speed-varying flywheel", that is a flywheel driven by a suitable cam-linkage mechanism. Demeulenaere and De Schutter [8] proposed a wide variety of input torque balancers, mainly based on inverted cam mechanisms and on a centrifugal pendulum guided by a fixed cam [9-11].

Also "potential balancers" have been presented. As an example, in papers $[12,13]$ a methodology is proposed to design a cam-spring balancer to compensate exactly the input torque variations.

It is interesting to point out that literature also proposed some input torque balancers that can be classified as "hybrid devices" or "active balancers". They are essentially two degrees of freedom mechanisms: the input shaft is driven by the main motor, while the auxiliary shaft is driven and controlled by a servomotor.

For instance, in Ref. [14] a quite complex planar seven-bar linkage is proposed as balancing device, while in Refs. $[15,16]$ a simple differential gear train is used as active balancer: one of its two input shafts is driven by a servomotor while the other shaft is connected to the follower.
It is worth to note that smoothing the input torque, or reducing the motor speed fluctuation, are related but not equivalent objectives. They are the same when the balancing mechanism is designed to suppress any torque fluctuation or to impose zero drive speed fluctuation [3].

When choosing or designing a balancer, the designer should take into consideration the kind of motor used to drive the whole system: if the drive exhibits a very high stiffness (e.g., a synchronous motor) the speed fluctuation is negligible and the goal is to minimize the required torque. Conversely, when the stiffness of the motor is low (e.g., a constant torque motor), the main balancer task should be the reduction of the speed variation.

\section{Dynamical Model}

With reference to Fig. 1, the system that will be analyzed in this paper is composed of the original 1-dof machine to be balanced and of two input torque balancing devices: $\mathrm{KB}$ and $\mathrm{PB}$ storing, respectively, kinetic and potential energy. Without loss of generality the original machine can be reduced to an equivalent inertia $J_{O}$ sensed by the main shaft, a supplied motor torque $M_{M}$ and an equivalent moment $M_{O}$ representing the loads acting on the system.

The two balancers can be considered as two degrees of freedom mechanisms and most of the input torque balancing techniques available in literature can be approached with this model. The main shaft of both
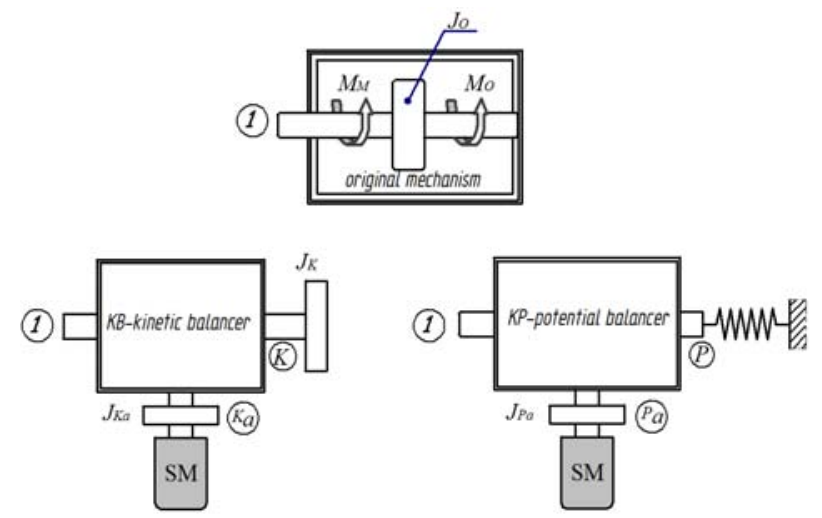

Fig. 1 Simplified model of the overall system. 
balancers, labeled with 1 in Fig. 1, is connected to a shaft of the original mechanism (usually the main shaft), while the auxiliary shaft $a$ can be controlled by a servomotor SM or held stationary (lowering the balancer dof's to one). The energy storage devices can be found at their output shafts $K$ and $P$.

Therefore, the complete system will have a maximum of three coordinates: the main shaft rotation $q_{1}$ and the rotations of the auxiliary shafts $q_{K_{a}}$ and $q_{P_{a}}$.

The dynamical model of the general 3-dof system will be derived using the Lagrangian formulation:

$$
\mathcal{F}_{i}=\frac{d}{d t}\left(\frac{\partial L}{\partial \dot{q}_{i}}\right)-\frac{\partial L}{\partial q_{i}} \quad i=1 . .3
$$

where $L=T-V$ is the Lagrangian of the system, $T$ is the kinetic energy, $V$ is the potential energy, and $\mathcal{F}_{i}$ represents the generalized force.

In order to simplify the mathematical model of the system some assumptions are made:

- friction forces are negligible;

- each moving part of the system is rigid and joint clearances are neglected;

- the generalized forces act only on the original system and depend only on the main shaft rotation $q_{1}$;

- the potential energy stored in the "kinetic balancer" as well as the kinetic energy in the "potential balancer" is both considered negligible.

\subsection{Dynamical Model of the Whole System}

The total kinetic energy of the system can be written as:

$$
T=\frac{1}{2} J_{O} \dot{q}_{1}^{2}+\left(\frac{1}{2} J_{K} \dot{q}_{K}^{2}+\frac{1}{2} J_{K_{a}} \dot{q}_{K_{a}}^{2}+\frac{1}{2} J_{P_{a}} \dot{q}_{P_{a}}^{2}\right)
$$

where $J_{O}$, which depends on $q_{1}$, is the variable moment of inertia of the original system sensed by the main shaft, $J_{K}$ is the constant moment of inertia of the output shaft of the "kinetic balancer". $J_{K_{a}}$ and $J_{P_{a}}$ are, respectively, the moments of inertia associated to the auxiliary shafts of the "kinetic" and "potential balancer".

Neglecting the potential energy of the original mechanism, the potential energy of the whole system depends only on the generalized coordinate $q_{P}$ : $V=V\left(q_{P}\right)$.

The kinematic relations among the first derivatives of the dependent coordinates $\left(q_{K}\right.$ and $\left.q_{P}\right)$ and the independent ones ( $q_{1}, q_{K a}$ and $\left.q_{P a}\right)$ can be obtained as:

$$
\dot{q}_{K}=\frac{\partial q_{K}}{\partial q_{1}} \dot{q}_{1}+\frac{\partial q_{K}}{\partial q_{K_{a}}} \dot{q}_{K_{a}}=f_{K_{1}} \dot{q}_{1}+f_{K_{a}} \dot{q}_{K_{a}}
$$

and

$$
\dot{q}_{P}=\frac{\partial q_{P}}{\partial q_{1}} \dot{q}_{1}+\frac{\partial q_{P}}{\partial q_{P_{a}}} \dot{q}_{P_{a}}=f_{P_{1}} \dot{q}_{1}+f_{P_{a}} \dot{q}_{P_{a}}
$$

The generalized transmission functions $f_{K_{1}}, f_{K_{a}}$, $f_{P_{1}}$ and $f_{P_{a}}$ are, in general, coordinate dependent.

Differentiating $L$ with respect to $q_{i}$ and $L$ with respect to $\dot{q}_{i}$ and time, we get the equations of motion of the whole system:

$$
\left\{\begin{array}{l}
M_{M}+M_{O}=\left(J_{O}+J_{K} f_{K_{1}}^{2}\right) \ddot{q}_{1}+J_{K} f_{K_{1}} f_{K_{a}} \ddot{q}_{K_{a}}+ \\
\left(J_{K} f_{K_{1}} \frac{\partial f_{K_{1}}}{\partial q_{1}}+\frac{1}{2} \frac{d J_{O}}{d q_{1}}\right) \dot{q}_{1}^{2}+ \\
J_{K}\left(f_{K_{1}} \frac{\partial f_{K_{a}}}{\partial q_{K_{a}}}-f_{K_{a}} \frac{\partial f_{K_{a}}}{\partial q_{1}}+f_{K_{a}} \frac{\partial f_{K_{1}}}{\partial q_{K_{a}}}\right) \dot{q}_{K_{a}}^{2}+ \\
2 J_{K} f_{K_{1}} \frac{\partial f_{K_{1}}}{\partial q_{K_{a}}} \dot{q}_{1} \dot{q}_{K_{a}}+\frac{d V}{d q_{P}} f_{P_{1}} \\
M_{K_{a}}=J_{K} f_{K_{1}} f_{K_{a}} \ddot{q}_{1}+\left(J_{K_{a}}+J_{K} f_{K_{a}}^{2}\right) \ddot{q}_{K_{a}}+ \\
J_{K}\left(f_{K_{1}} \frac{\partial f_{K_{a}}}{\partial q_{1}}+f_{K_{a}} \frac{\partial f_{K_{1}}}{\partial q_{1}}-f_{K_{1}} \frac{\partial f_{K_{1}}}{\partial q_{K_{a}}}\right) \dot{q}_{1}^{2}+ \\
J_{K} f_{K_{a}} \frac{\partial f_{K_{a}}}{\partial q_{K_{a}}} \dot{q}_{K_{a}}^{2}+2 J_{K} f_{K_{a}} \frac{\partial f_{K_{a}}}{\partial q_{1}} \dot{q}_{1} \dot{q}_{K_{a}} \\
M_{P_{a}}=J_{P_{a}} \ddot{q}_{P_{a}}+\frac{d V}{d q_{P}} f_{P_{a}}
\end{array}\right.
$$

where, $M_{M}\left(q_{1}, \dot{q}_{1}\right)$ is the main motor supplied torque, $M_{O}\left(q_{1}\right)$ represents the loads acting on the original machine, while $M_{K_{a}}$ and $M_{P_{a}}$ are the torque supplied by the auxiliary motors.

\section{Synthesis of 1-dof Balancer}

In this section we analyze the case of the original machine input torque balancing by means of a 1-dof balancer, potential and kinetic.

The equation of motion can be readily obtained from the first equation of system Eq. (4) assuming 
$\dot{q}_{K_{a}} \equiv 0$ and $\dot{q}_{P_{a}} \equiv 0$ :

$$
\begin{aligned}
M_{M}+M_{O}= & \left(J_{O}+J_{K} f_{K_{1}}^{2}\right) \ddot{q}_{1}+ \\
& \left(J_{K} f_{K_{1}} \frac{d f_{K_{1}}}{d q_{1}}+\frac{1}{2} \frac{d J_{O}}{d q_{1}}\right) \dot{q}_{1}^{2}+\frac{d V}{d q_{P}} f_{P_{1}}
\end{aligned}
$$

In this case the overall system becomes a 1-dof mechanism.

In order to derive the mathematical model that describes the steady-state we impose a constant operating speed $\dot{q}_{1}=\omega_{0}$, thus $\ddot{q}_{1} \equiv 0$.

This condition could be obtained using a stiff motor (e.g., a synchronous motor) without any balancer: in this case the speed fluctuation would be negligible with the motor torque $M_{M}$ varying periodically.

Nevertheless, we imagine that the main motor only supplies a constant torque, to sustain the periodic motion, while the balancer absorbs the whole load variation. The required motor torque $M_{M}$ is determined as follows:

$$
\bar{M}_{M}=-\frac{1}{\Phi} \int_{0}^{\Phi} M_{O}\left(q_{1}\right) d q_{1}
$$

where $\Phi$ is the angular period (generally $2 \pi$ ).

With the above assumptions the design equation becomes:

$$
\left(J_{K} f_{K_{1}} \frac{d f_{K_{1}}}{d q_{1}}+\frac{1}{2} \frac{d J_{O}}{d q_{1}}\right) \omega_{0}^{2}+\frac{d V}{d q_{P}} f_{P_{1}}=\bar{M}_{M}+M_{O}
$$

In case of no energy dissipation or no external load, $\bar{M}_{M}$ vanishes. Further simplification can be obtained considering the presence of only one kind of balancer (kinetic or potential).

\subsection{Synthesis of 1-dof Kinetic Balancer}

When only a "kinetic balancer" is used for compensating the torque/speed fluctuations, see for example Ref. $[4,5,7,9,11]$, the design goal will be to determine a suitable value of $J_{K}$ and the corresponding optimal transmission function $f_{K_{1}}$. In this case the potential energy of the system can be neglected, hence Eq. (7) simplifies as follow:

$$
\left(J_{K} f_{K_{1}} \frac{d f_{K_{1}}}{d q_{1}}+\frac{1}{2} \frac{d J_{O}}{d q_{1}}\right) \omega_{0}^{2}=\bar{M}_{M}+M_{O}
$$

Rearranging the above equation we get:

$$
J_{K} \omega_{0}^{2} f_{K_{1}} \frac{d f_{K_{1}}}{d q_{1}}=\bar{M}_{M}+M_{O}-\frac{1}{2} \frac{d J_{O}}{d q_{1}} \omega_{0}^{2}
$$

Integrating with respect to $q_{1}$ we obtain:

$$
\begin{aligned}
& \frac{1}{2} J_{K} \omega_{0}^{2}\left[f_{K_{1}}^{2}\left(q_{1}\right)-f_{K_{1}}^{2}(0)\right]= \\
& \int_{0}^{q_{1}}\left(\bar{M}_{M}+M_{O}-\frac{1}{2} \frac{d J_{O}}{d q_{1}} \omega_{0}^{2}\right) d q_{1}
\end{aligned}
$$

Posing:

$$
\begin{aligned}
& \mathcal{E}\left(q_{1}\right)=\int_{0}^{q_{1}}\left(\bar{M}_{M}+M_{O}\right) d q_{1}- \\
& \frac{1}{2}\left[J_{O}\left(q_{1}\right)-J_{O}(0)\right] \omega_{0}^{2}
\end{aligned}
$$

we have:

$$
\frac{1}{2} J_{K} \omega_{0}^{2}\left[f_{K_{1}}^{2}\left(q_{1}\right)-f_{K_{1}}^{2}(0)\right]=\mathcal{E}\left(q_{1}\right)
$$

or in more compact form:

$$
\frac{1}{2} J_{K} \omega_{0}^{2} f_{K_{1}}^{2}=\mathcal{E}\left(q_{1}\right)+\mathcal{C}
$$

where $\mathcal{C}=\frac{1}{2} J_{K} \omega_{0}^{2} f_{K_{1}}^{2}(0)$ is an arbitrary constant (it can be thought as the kinetic energy of the balancer for $q_{1}=0$ ).

Solving for $f_{K_{1}}$ we obtain:

$$
f_{K_{1}}=\sqrt{\frac{2\left[\mathcal{E}\left(q_{1}\right)+\mathcal{C}\right]}{J_{K} \omega_{0}^{2}}}
$$

With this kinetic approach a problem arises because, generally, it is impossible to realize the required transmission function with a reciprocating mechanism, for example an ordinary cam-follower system, and more complicated mechanisms must be used.

As a matter of fact, the integral of $f_{K_{1}}$ over the period of the main shaft cannot be null.

$$
\int_{0}^{2 \pi} \sqrt{\frac{2\left[\mathcal{E}\left(q_{1}\right)+\mathcal{C}\right]}{J_{K} \omega_{0}^{2}}} d q_{1}=\Psi
$$

If $\Psi$ is chosen as $\Psi=2 \pi$, the required $f_{K_{1}}$ can be exactly obtained using a complex mechanisms $[5,6$, 8, 9]. Alternatively, an approximate solution, requiring a lower synthesis effort, can be adopted as proposed in Refs. $[17,18]$. 
Solving for $J_{k}$, we obtain the theoretical value which can exactly balance the irregularities:

$$
\bar{J}_{K}=\frac{1}{2 \pi^{2}}\left[\int_{0}^{2 \pi} \sqrt{\frac{\varepsilon\left(q_{1}\right)+\mathcal{C}}{\omega_{0}^{2}}} d q_{1}\right]^{2}
$$

Eventually, the theoretical optimal value of $f_{K_{1}}$ is computed using Eq. (12).

It is worth to observe that the effect of $\mathcal{C}$ is to raise the value of $\bar{J}_{K}$, conversely higher values of $\bar{J}_{K}$ imply $f_{K_{1}}$ closer to unity.

An advantage of this device is that it can balance the required torque for any assigned speed, providing that the original system is purely inertial. In fact, in this case, $M_{O}\left(q_{1}\right)$ and $M_{M}$ vanish, thus the optimal value of $f_{K_{1}}$ does not depend on $\omega_{0}$, as inferable from Eqs. (14) and (17). In other words, the kinetic energy of the whole system does not fluctuate for any nominal speed.

On the contrary, when the inertial contribution on the input torque is trivial compared to the external work, the "kinetic balancer" is optimal just for the design speed.

\subsection{Synthesis of 1-dof Potential Balancer}

When a "potential balancer" is used to reduce the torque/speed fluctuations, the design equation can be obtained from Eq. (7) imposing $J_{K}=0$, hence:

$$
\omega_{0}^{2}\left(\frac{1}{2} \frac{d J_{O}}{d q_{1}}\right)+\frac{d V}{d q_{P}} f_{P_{1}}=\bar{M}_{M}+M_{O}
$$

Rearranging the above equation and noting that in this case $f_{P_{1}}=\frac{d q_{P}}{d q_{1}}$ ( $q_{P}$ depends only on $q_{1}$ ) we get:

$$
\frac{d V}{d q_{1}}=\bar{M}_{M}+M_{O}-\frac{1}{2} \frac{d J_{O}}{d q_{1}} \omega_{0}^{2}
$$

and integrating with respect to $q_{1}$ we obtain:

$$
V\left(q_{1}\right)-V(0)=\varepsilon\left(q_{1}\right)
$$

Thus, the potential energy variation must compensate for the variation in kinetic energy of the original system and for the work done by the forces acting on it.

Choosing a suitable value of potential energy evaluated for $q_{1}=0$ and naming it with $\mathcal{C}_{P}$, we get:

$$
V\left(q_{1}\right)=\mathcal{E}\left(q_{1}\right)+\mathcal{C}_{P}
$$

Up to now, no assumption has been made concerning the kind of potential energy $V$. As an example, as reported in Refs. [12, 13], assuming elastic potential energy, $V$ can be written as: $V=\frac{1}{2} k q_{P}^{2}$, where $k$ is the spring stiffness.

Once the appropriate spring constant $\bar{k}$ is selected, $q_{P}\left(q_{1}\right)$ can be determined from Eq. (18), namely:

$$
q_{P}\left(q_{1}\right)=\sqrt{\frac{2\left[\mathcal{E}\left(q_{1}\right)+\mathcal{C}_{P}\right]}{\bar{k}}}
$$

The main advantage of this balancer is that it can be realized using a reciprocating mechanism, like an ordinary cam-follower system, where a spring is connected to the follower, moreover, when the generalized forces acting on the machine depend only on the main shaft rotation and the inertia forces are negligible, the balancing results are independent of the drive speed.

On the other hand, when the inertia loads are dominant, it is optimal just for the design drive speed $\omega_{0}$. The speed range of the PB can be extended using tunable stiffness springs: if the spring constant can be adjusted in order to have $k(\omega)=\bar{k}\left(\frac{\omega}{\omega_{0}}\right)^{2}$, the balancing results are optimal for any $\omega$. This statement can be proved noting that the second member of Eq. (19) depends only on $q_{1}$ : in fact also $\mathcal{C}_{P}$, the potential energy for $q_{1}=0$, depends quadratically on the ratio $\left(\frac{\omega}{\omega_{0}}\right)$, hence $q_{P}\left(q_{1}\right)$ remains unchanged for any speed.

In ideal condition, these considerations suggest using a "kinetic balancer" along with a "potential" one in order to exactly balance the input torque for any operating drive speed.

\section{Example: Design of a Balancer for a Scotch Yoke Mechanism}

In this section we present an application of this 
framework to the balancing of a 1-dof system, to show how this general method fits in a real case. The system is kept as simple as possible to avoid dispersion on kinematic details and to focus on the application of the method.

The system is composed of a gear motor (with a constant torque-speed characteristic) that is coupled directly to the crank of a Scotch yoke (Fig. 2).

Only an external load is considered: a force $F_{R}$, function of slider displacement, applied on the slider itself. Only the crank and the slider possess inertia. Members' weight can be neglected, since the slider moves horizontally and the crank is generally balanced.

The main data of the system are reported in Table 1.

Indicating with $\mathrm{q}_{1}$ the angle of rotation of the crank, the slider displacement $x$, velocity $\dot{x}$ and acceleration $\ddot{x}$, are readily obtained:

$$
\begin{aligned}
& x=r\left(1-\cos \left(q_{1}\right)\right) \\
& \dot{x}=r \dot{q}_{1} \sin \left(q_{1}\right) \\
& \ddot{x}=r \ddot{q}_{1} \sin \left(q_{1}\right)+r \dot{q}_{1}{ }^{2} \cos \left(q_{1}\right)
\end{aligned}
$$

The external force $F_{R}$ acts on the slider during the working stroke and has the following expression:

$$
F_{R}(x)=\frac{F_{0}}{2}\left(1-\cos \left(\frac{2 \pi}{c} x\right)\right)
$$

with $\mathrm{F}_{0}=2,000 \mathrm{~N}$.

The equivalent inertia $J_{O}$ sensed by the main shaft in this case becomes:

$$
J_{O}\left(q_{1}\right)=J_{c}+m\left(r \sin \left(q_{1}\right)\right)^{2}
$$

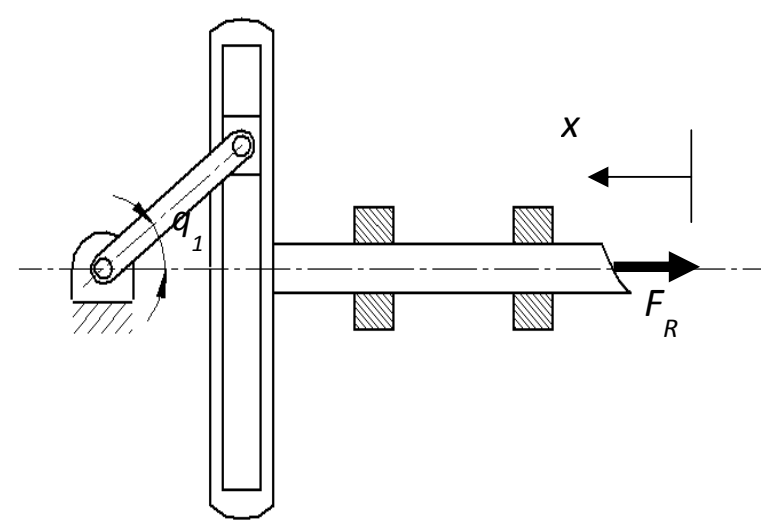

Fig. 2 Sketch of the scotch yoke mechanism.
Table 1 System parameters.

\begin{tabular}{ll}
\hline Crank radius $r$ & $0.1 \mathrm{~m}$ \\
Stroke length $c$ & $0.2 \mathrm{~m}$ \\
Slider mass $m$ & $40 \mathrm{~kg}$ \\
Crank inertia $\mathrm{J}_{\mathrm{C}}$ & $0.1 \mathrm{kgm}^{2}$ \\
Angular velocity $n_{\mathbf{0}} / \mathbf{0}$ & $200 \mathrm{rpm} / 20.94 \mathrm{rad} / \mathrm{s}$ \\
\hline
\end{tabular}

The load $M_{O}\left(q_{1}\right)$ acting on the system, considering a constant crank angular velocity $\omega_{0}$, is:

$$
M_{O}\left(q_{1}\right)=F_{r}(x) r \sin \left(q_{1}\right)
$$

The average motor torque $\bar{M}_{M}$ can be calculated as follows:

$$
\bar{M}_{M}=\frac{1}{2 \pi} \int_{0}^{2 \pi} M_{O}\left(q_{1}\right) d q_{1} \cong 31.83 \mathrm{Nm}
$$

The energy variation $\mathcal{E}\left(q_{1}\right)$ (see Eq. (10)) can be numerically obtained and its graph is shown in Fig. 3.

Having all involved quantities a common period of $2 \pi$, corresponding to a revolution of the crank, analyis is limited to the interval $0 \leq q_{1} \leq 2 \tilde{\pi}$.

\subsection{Kinetic Balancer}

In this synthesis the constant $C$, needed to make energy always positive (see Eq. (11)), can be assumed arbitrarily. A value $20 \%$ greater than the absolute minimum value of $\mathcal{E}\left(q_{1}\right)$ (i.e., $C=1.2\left|\mathcal{E}\left(q_{1}\right)\right| \cong$ $220 \mathrm{~J}$ ) has been chosen.

Applying Eq. (14) the required inertia $\bar{J}_{K}$ of the flywheel can be calculated: for this simple system we get $\bar{J}_{K} \cong 0.53 \mathrm{kgm}^{2}$.

Now, from Eq. (12), the optimal transmission function $f_{K_{1}}$ can be numerically obtained (see Fig. 4) and a mechanism (i.e., double-crank mechanism, non-circular gears) connecting the flywheel to the main shaft can be synthesized.

A multi-body model, within MSCAdams, has been built to test the dynamic behavior both of the original and balanced machine. To keep the model simple, the transmission function of the balancer has been reproduced with an Adams "general constraint". With the balancer and considering a constant torque motor, the crank angular speed remains constant, showing therefore a zero periodic irregularity (Fig. 5). Without 


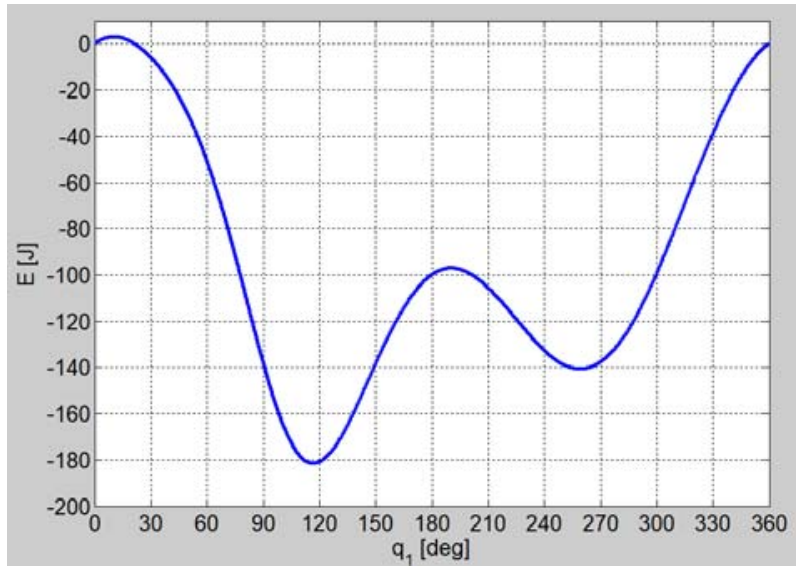

Fig. 3 Energy variation $\mathcal{E}\left(q_{1}\right)$ at constant speed $\omega_{0}$. Reference point is taken at the beginning of a cycle $\left(q_{1}=0\right)$.

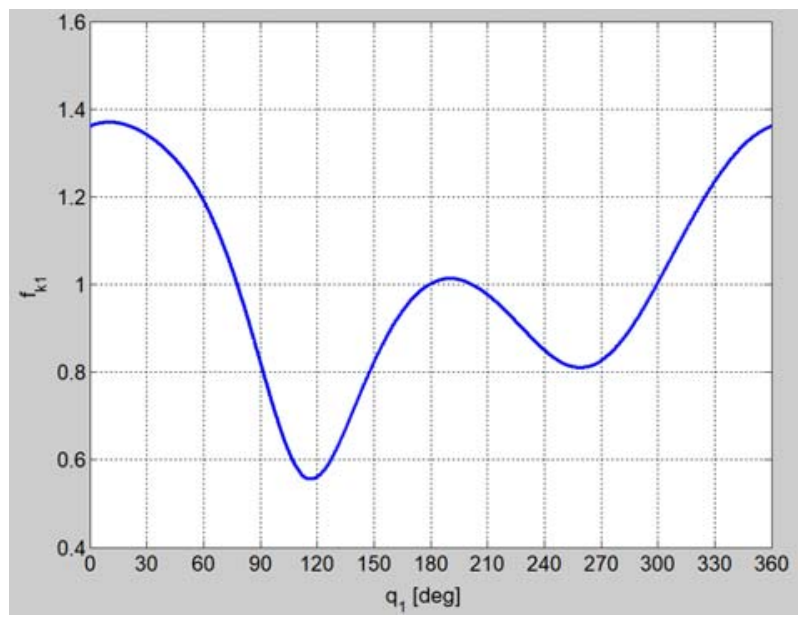

Fig. 4 Optimal transmission function $\mathrm{f}_{\mathrm{K} 1}\left(\mathrm{q}_{1}\right)$.

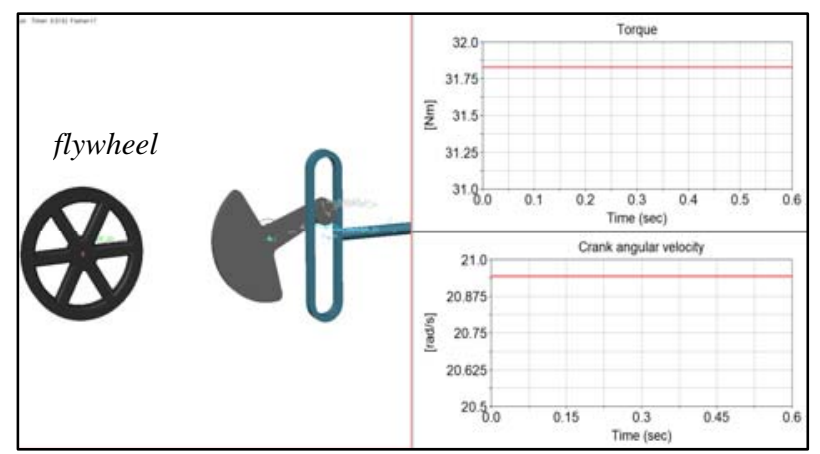

Fig. 5 Adams model of the original system with the Kinetic Balancer (left). Applied torque and resulting crank speed (right).

the balancer, speed fluctuates with a periodic irregularity of about $42 \%$.

\subsection{Potential Balancer}

The constant $C_{P}$ has been chosen as in the previous case, thus the required potential energy $\mathrm{V}\left(q_{1}\right)$ (see Eq. (17)) corresponds to $\mathcal{E}\left(q_{1}\right)$, shifted upwards by constant $C_{P}$.

Assuming an elastic potential energy $V$ (see Section 3.2), a suitable value for the spring stiffness can be selected. In this example we impose the cam rise $(\mathrm{h}=$ $0.03 \mathrm{~m})$.

Recalling Eq. (19): $q_{P}\left(q_{1}\right)=\sqrt{\frac{2\left[\mathcal{E}\left(q_{1}\right)+\mathcal{C}_{P}\right]}{k}}$ and noting that $h=q_{P M A X}-q_{P M I N}$, the required stiffness can be obtained as follow:

$$
k=\left(\frac{\sqrt{2\left[\varepsilon_{M A X}+\mathcal{C}_{P}\right]}-\sqrt{2\left[\mathcal{E}_{M I N}+\mathcal{C}_{P}\right]}}{h}\right)^{2} \cong 173,300 \mathrm{~N} / \mathrm{m} .
$$

Thus the displacement of the output of the potential balancer $q_{P}\left(q_{1}\right)$ can be determined. The loading of the spring can be obtained by means of a cam mechanism: as a matter of fact the $q_{p}$ corresponds to the displacement function of the cam (Fig. 6).

For this case too, a multi-body model of the machine has been built and simulated as shown in Fig. 7.

Here a cam, synthesized using the determined $q_{\mathrm{P}}$ and coupled directly to the crank shaft, moves the spring. Considering a constant torque motor and the presence of the balancer, the crank angular speed remains constant, showing therefore a zero periodic irregularity. Obviously, without the balancer, speed fluctuation is the same as before.

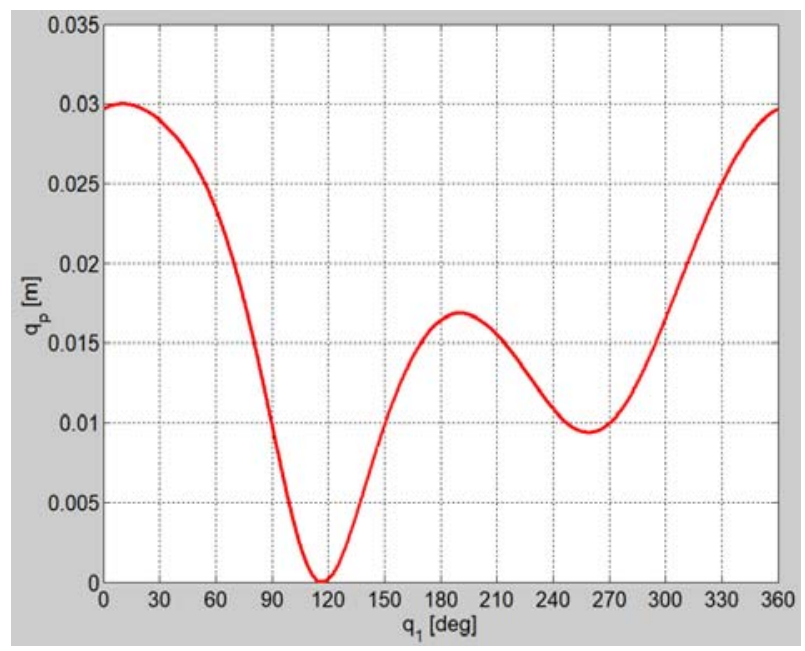

Fig. 6 Required displacement of the follower. 


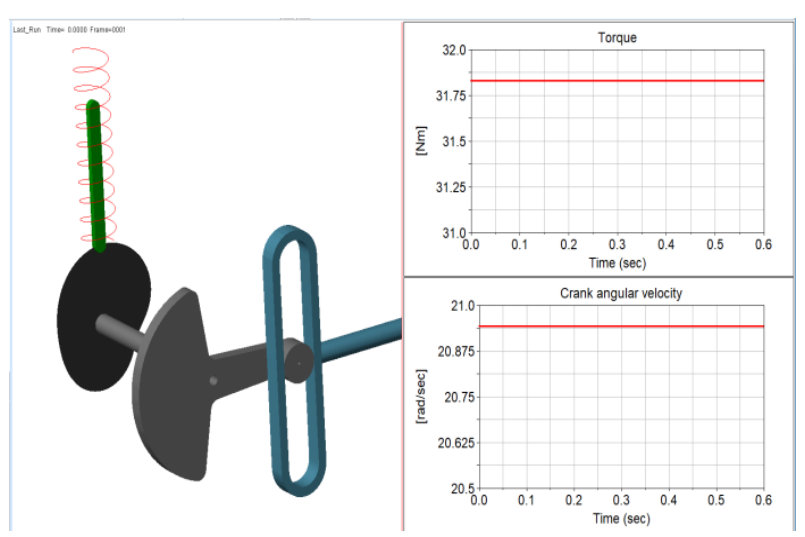

Fig. 7 Adams model of the original system with the Potential Balancer (left). Applied torque and resulting crank speed (right).

\section{Conclusions}

In this paper a general methodology to synthesize a balancing mechanism for a 1-dof machine, subjected to both inertial and periodic loads, has been presented. The general equations of motion for the machine coupled with a 2-dof or 1-dof balancing system have been stated. A simple application, consisting in the balancing of a 1-dof machine with a potential/kinetic balancer, has been worked out for a better comprehension of the proposed procedure. Balancers synthesis has been performed using a dedicated Matlab program and verification tests on the whole assembly (original machine plus balancer) have been performed with MSC Adams. The simulations show a perfect balancing in case the machine is working in nominal condition. Small speed/torque fluctuations are present if only one kind of balancer is used and the working speed is different from the design one. Simulations show also that the adoption of both a potential and kinetic balancer allows keeping the machine balanced for different working speeds. A complete sensitivity analysis, regarding masses, transmission rations and forces (machine parameters) will be a possible next step.

With regard to this, 2-dof balancing systems, with constant transmission ratios, have the potential to be easily tuned to the actual parameters and exercise loads of the machine. Such differential devices could be quite easily fitted to an existing machine, with only minor adjustments and, if required, directly on the "disturbing" shaft. This approach, that seems quite promising from a theoretical point of view, should be tested against real machines including their vibrational characteristics, backlashes, friction, in any case the law of motion of the auxiliary motor could be optimized to avoid potentially dangerous harmonics or torque reversing rather than to obtain torque balancing.

As a further development a 2-dof balancer (KB or $\mathrm{PB})$ with a variable transmission function could allow also the synthesis of a simple and inexpensive balancing mechanism, yielding an approximate value of $f_{K_{1}}$ or $f_{P_{1}}$, leaving to the auxiliary motor the task to balance the residual torque fluctuations, or to cope with exercise loads differently from the design ones.

\section{References}

[1] Lowen, G. G., and Berkof, R. S. 1968. "Balancing of Linkages.” Journal of Mechanisms 3 (4): 221-31.

[2] Arakelian, V., and Briot, S. 2015. "Balancing of Linkages and Robot Manipulators." Mechanisms and Machine Science 27.

[3] Demeulenaere, B., and Berkof, R. 2008. "Improving Machine Drive Dynamics: A Structured Design Approach toward Balancing." ASME J. Mech. Des. 130 (8): 082302-1-082302-9.

[4] Kochey, J. 1990. "General Method for Active Balancing of Combined Shaking Moment and Torque Fluctuation in Planar Linkage." Mechanism and Machine Theory 25 (6): 679-87.

[5] Dooner, D. B. 1997. "Use of Noncircular Gears to Reduce Torque and Speed Fluctuations in Rotating Shafts.” Journal of Mechanical Design 119 (2): 299-306.

[6] Yao, Y., and Yan, H. 2003. "A New Method for Torque Balancing of Planar Linkages Using Non-circular Gears." In Proceedings of the Institution of Mechanical Engineers, Part C: Journal of Mechanical Engineering Science 217 (5): 495-503.

[7] Guilan, T., Haibo, F., and Weiyi, Z. 1999. "A New Method of Torque Compensation for High Speed Indexing Cam Mechanism." Journal of Mechanical Design 121 (2): 319-23.

[8] Demeulenaere, B., and De Schutter, J. 2005. "Input Torque Balancing Using an Inverted Cam Mechanism." Journal of Mechanical Design 127 (5): 887-900.

[9] Demeulenaere, B., Spaepen, P., and De Schutter, J. 2005. "Input Torque Balancing Using a Cam-Based Centrifugal 
Pendulum: Design Procedure and Example.” Journal of Sound and Vibration 283 (1): 1-20.

[10] Demeulenaere, B., Spaepen, P., and De Schutter, J. 2005. "Input Torque Balancing Using a Cam-Based Centrifugal Pendulum: Design Optimization and Robustnesss." Journal of Sound and Vibration 283 (1): 21-46.

[11] Demeulenaere, B., Spaepen, P., Masselis, S., Cornelissen, P., Pinte, G., Hemelsoen, J., Boonen, R., Roelstraete, K., Desmet, W., Swevers, J., and De Schutter, J. 2008. "Experimental Validation of Input Torque Balancing Applied to Weaving Machinery." Journal of Mechanical Design 130 (2): 1-10.

[12] WU, C. J., and Angeles, J. 2001. "The Optimum Synthesis of an Elastic Torque-Compensating Cam Mechanism." Mechanism and Machine Theory 36 (2): 245-59.

[13] Arakelian, V., and Briot, S. 2010. "Simultaneous Inertia Force/Moment Balancing and Torque Compensation of Slider-Crank Mechanisms.” Mechanics Research
Communications 37 (2): 265-9.

[14] Greenough, J., Bradshaw, W., Gilmartin, M., Douglas, S., and Jones, J. 1995. "Design of Hybrid Machines." In Proceedings of Ninth World Congress on the Theory of Machines and Mechanisms, 2501-5.

[15] JING, S., and Yan-An, Y. 2009. "An Independent Active Torque Balancer Using a Servo-Controlled Differential Gear Train." Transactions of the Canadian Society for Mechanical Engineering 33 (2): 32-48.

[16] Jing, S., and Yan-An, Y. 2011. "An Active Gear Balancer for Torque Compensation." Journal of Mechanical Design 133 (1): 014502-1-014502-10.

[17] Lorenzi, V., Riva, R., Strada, R., and Zappa, B. 2004. "On Using Mini-flywheels to Reduce Speed Fluctuations." In Proceedings of the 11th World Congress in Mechanism and Machine Science, 622-7.

[18] Zappa, B., Lorenzi, V., Righettini, P., and Strada, R. 2016. "An Intuitive Approach to the Kinematic Synthesis of Mechanisms." In Proceedings of ICERI 2016, 6888-96. 